Gut, 1987, 28, 668-674

\title{
Hepatic sarcomas in adults: a review of 25 cases
}

\author{
A FORBES, B PORTMANN, P JOHNSON, AND ROGER WILLIAMS \\ From the Liver Unit, King's College School of Medicine and Dentistry, Denmark Hill, London
}

SUmmary Twenty five patients with an apparently primary sarcoma of the liver are reviewed. Presenting complaints were non-specific, but hepatomegaly and abnormal liver function tests were usual. Use of the contraceptive pill (four of 11 women) was identified as a possible risk factor; one patient had previously been exposed to vinyl chloride monomer. Detailed investigation showed that the primary tumour was extrahepatic in nine of the 25 patients. Distinguishing features of the 15 patients with confirmed primary hepatic sarcoma included a lower incidence of multiple hepatic lesions and a shorter time from first symptoms to diagnosis, but the most valuable discriminator was histology. Angiosarcomas and undifferentiated tumours were all of hepatic origin, epithelioid haemangioendotheliomas (EHAE) occurred as primary and secondary lesions and all other differentiated tumours arose outside the liver. The retroperitoneum was the most common site of an occult primary tumour and its careful examination therefore crucial: computed tomography scanning was found least fallible in this respect in the present series. Where resection (or transplantation), the best treatment, was not possible, results of therapy were disappointing, prognosis being considerably worse for patients with primary hepatic tumours. Patients with EHAE had a better overall prognosis regardless of primary site.

Malignant mesenchymal tumours arising primarily in the liver are extremely rare in adults ${ }^{12}$ and little less so in children. Angiosarcoma (or malignant haemangioendothelioma) has a recognised association with Thorotrast, ${ }^{+}$arsenic $^{5}$ and vinyl chloride monomer," but no definite aetiological factors have been identified for the other varieties of hepatic sarcoma. Ishak has reviewed the pathology of adult tumours' but with a single exception" clinical reports comprise, at most, four cases or are literature reviews. Features distinguishing primary from secondary hepatic sarcoma have been little considered. In the present study we have reviewed 25 adults referred to this unit between 1970) and 1985 with an apparent primary hepatic sarcoma. After further investigation it was considered more likely that in nine cases the hepatic lesion was the result of metastic spread from a primary tumour elsewhere; the relevance of this to treatment and prognosis is considered.

\section{Methods \\ PATIENTS \\ The 25 patients (14 male) were caucasian with a \\ Address for correypondence: Dr A Forbes. I iver l !nit. King sc college Hospital. Denmarth Hill. Iondon S1:5 GRS. \\ Receiscd for publication I9 Soptember leso.}

median age of 41 years (range 15-69 years). Referral was usually for consideration of resection or orthotopic transplantation but in seven cases no diagnosis had been reached at the time of referral although hepatic tumour was suspected. Hepatic tissue was obtained by percutaneous liver biopsy except in the first case which was a necropsy diagnosis and in cases 2,22 , and 25 where biopsies were taken at laparotomy at the referring hospital. Histology was reviewed for this report with diagnoses of sarcoma type made according to accepted criteria.' The sarcomatous nature of undifferentiated tumours was supported by positive cell staining with the antivimentin monoclonal antibody ${ }^{x}$ and/or by negative staining for epithelial cytoskeleton (CAM 5.2) (as sarcomas are non-epithelial tumours). Antivimentin was from Dako Ltd, and CAM 5.2 a gift from Imperial Cancer Research Fund Laboratories. Epithelioid haemangioendothelioma (EHAE)" was diagnosed on the basis of typical histological appearances and on the demonstration of factor VIII associated antigen within tumour cell cytoplasm" using the monoclonal antibody from Dako Ltd. Exploratory laparotomy was carried out before referral in five patients (cases 2,7,14,22, and 25) and in only three patients (cases 13,15, and 18) was careful open examination of the abdomen not undertaken, either at laparotomy or at necropsy. 


\section{Results}

CI.INICAI

Presenting complaints included nausea, general malaise, right upper quadrant abdominal pain and weight loss at some time in all patients. Eight patients described abdominal swelling and five had sweats or fevers. Case 18 also had a two year history of hip pain. All but two patients had hepatomegaly (median $8 \mathrm{~cm}$ palpable; range 2-20) and three had splenomegaly (cases 4, 6, and 23). Eight patients had ascites and seven were jaundiced. In only one case were liver function tests normal (case 11); alkaline phosphatase concentrations were at least twice the upper limit of normal in 20 cases and aminotransferases at least twice normal in eight. Seven patients had normochromic normocytic anaemia. No positive markers for hepatitis B (22 patients) nor abnormal concentrations of alphafetoprotein (22 patients) were recorded. Isotope and/or ultrasound liver scans showed filling defects in all cases, which were single in 12 cases and multiple in 13 (Table 1) (although an initially single lesion in case 25 became multifocal). Hepatic angiography in 16 patients showed hyper-

Table 1 Clinical and imaging features

\begin{tabular}{|c|c|c|c|c|c|c|}
\hline Patient & Age & Sex & $\begin{array}{l}\text { Unifocal } \\
\text { hepatic } \\
\text { lesion }\end{array}$ & $\begin{array}{l}\text { Hyper- } \\
\text { alvascular } \\
\text { arterio- } \\
\text { graphy }\end{array}$ & $\begin{array}{l}\text { Site of } \\
\text { primary }\end{array}$ & Histology \\
\hline 1 & 47 & $\mathrm{~F}$ & + & NA & Liver & AS \\
\hline 2 & 66 & $\mathrm{M}$ & - & NA & Vena cava & LMS \\
\hline 3 & 69 & $M$ & + & + & Colon & LMS \\
\hline 4 & 37 & M & + & NA & Liver & AS \\
\hline 5 & 58 & $\mathrm{~F}$ & - & + & Retroperitoncum & LMS \\
\hline 6 & 49 & $\mathrm{M}$ & - & - & Liver & AS \\
\hline 7 & 22 & $\mathrm{~F}$ & + & + & Liver & AS \\
\hline 8 & 54 & M & + & NA & Retroperitoncum & LMS \\
\hline 9 & 56 & $\mathrm{M}$ & - & + & Retroperitoncum & LMS \\
\hline 10 & 22 & $\mathrm{~F}$ & + & + & Liver & US \\
\hline 11 & 69 & $\mathrm{M}$ & + & NA & Liver & US \\
\hline 12 & 23 & $\mathrm{M}$ & - & NA & Liver & AS \\
\hline 13 & 41 & $\mathrm{~F}$ & - & NA & Retroperitoncum & EHAE \\
\hline 14 & 32 & $\mathrm{~F}$ & - & + & Stomach & LMS \\
\hline 15 & 44 & $\mathrm{~F}$ & - & NA & Retroperitoncum & MHPC \\
\hline 16 & 15 & $\mathrm{~F}$ & + & + & Liver & US \\
\hline 17 & 49 & $\mathrm{~F}$ & + & NA & Liver & US \\
\hline 18 & 32 & $\mathrm{~F}$ & - & + & Bonc & EHAE \\
\hline 19 & 37 & $\mathbf{M}$ & - & + & Liver & AS \\
\hline 20 & 38 & M & + & - & Retroperitoncum & MFIIC \\
\hline 21 & 22 & $\mathrm{M}$ & - & + & Liver & EHAE \\
\hline 22 & 19 & M & - & + & Liver & EHAE \\
\hline 23 & 54 & $\mathbf{M}$ & - & + & Liver & AS \\
\hline 24 & 30 & $\mathrm{~F}$ & + & + & Liver & FHAE \\
\hline 25 & 55 & $\mathrm{M}$ & + & + & Liver & AS \\
\hline
\end{tabular}

AS: angiosarcoma: EHAE: epithelioid hacmangioendothelioma: LMS: leiomyosarcoma: MHPC: malignant hacmangiopericytoma: MFHC: malignant fibrous histiocytoma; US: undifferentiated sarcoma. vascular lesions with abnormal displaced vessels similar to those seen in primary hepatocellular carcinoma in 14 cases; in two patients the apparent tumour mass was avascular. In all patients the retroperitoneum was examined by at least two techniques.

\section{HISTOLOGY}

Final histological diagnoses (Table 2) included eight angiosarcomas, six leiomyosarcomas, five epithelioid haemangioendotheliomas (Fig. 1a) and four undifferentiated sarcomas (Fig. 2). Staining for factor VIII associated antigen was positive in all cases of EHAE (Fig. 1b), and in the seven cases of angiosarcoma for which unstained sections remained available. In three of the angiosarcomas there was associated cirrhosis: the presence of hyaline inclusions implicated alcohol in two.

\section{AETIOLOGY}

With respect to potential aetiological factors, there was a history of industrial exposure to vinyl chloride monomer for nine months, five years before presentation, in case 19 (with angiosarcoma); occupational history was unremarkable in the remainder. with no patient exposed to arsenic nor Thorotrast. Two patients drank more than $400 \mathrm{~g}$ ethanol per week (cases 9 and 20) and 11 were smokers. Four women (cases 1, 14, 18, and 24) had taken the combined oral contraceptive for $4,8,11$, and 7 years respectively. Cases 3,9 , and 11 had a past history of malaria. A past history of neoplastic diseases was obtained for three patients. Case 3 had a symptomatic colonic tumour thought to be a benign leiomyoma resected four years before presentation; case 5 had a benign cystic tumour of the ovary resected 22 years before; case 11 had an anterior resection for colonic carcinoma eight years earlier. The brother of case 12 died at 26 years with a clinical diagnosis of primary liver tumour.

\section{Differentiation of primary from secondary hepatic sarcoma}

Of 25 patients with apparently localised primary hepatic sarcoma, nine were shown after detailed

\section{Table 2 Histological diagnoses}

\begin{tabular}{ll}
\hline Angiosarcoma & 8 (3 cirrhotic) \\
Leiomyosarcoma & 6 \\
Epithelioid hacmangiocndothelioma & 5 \\
Malignant hacmangiopericytoma & 1 \\
Mallignant fibrous histiocytoma & 1 (fibrosis, hyaline + \\
Undifferentiated sarcoma & 4 fatly change) \\
Total & 45
\end{tabular}




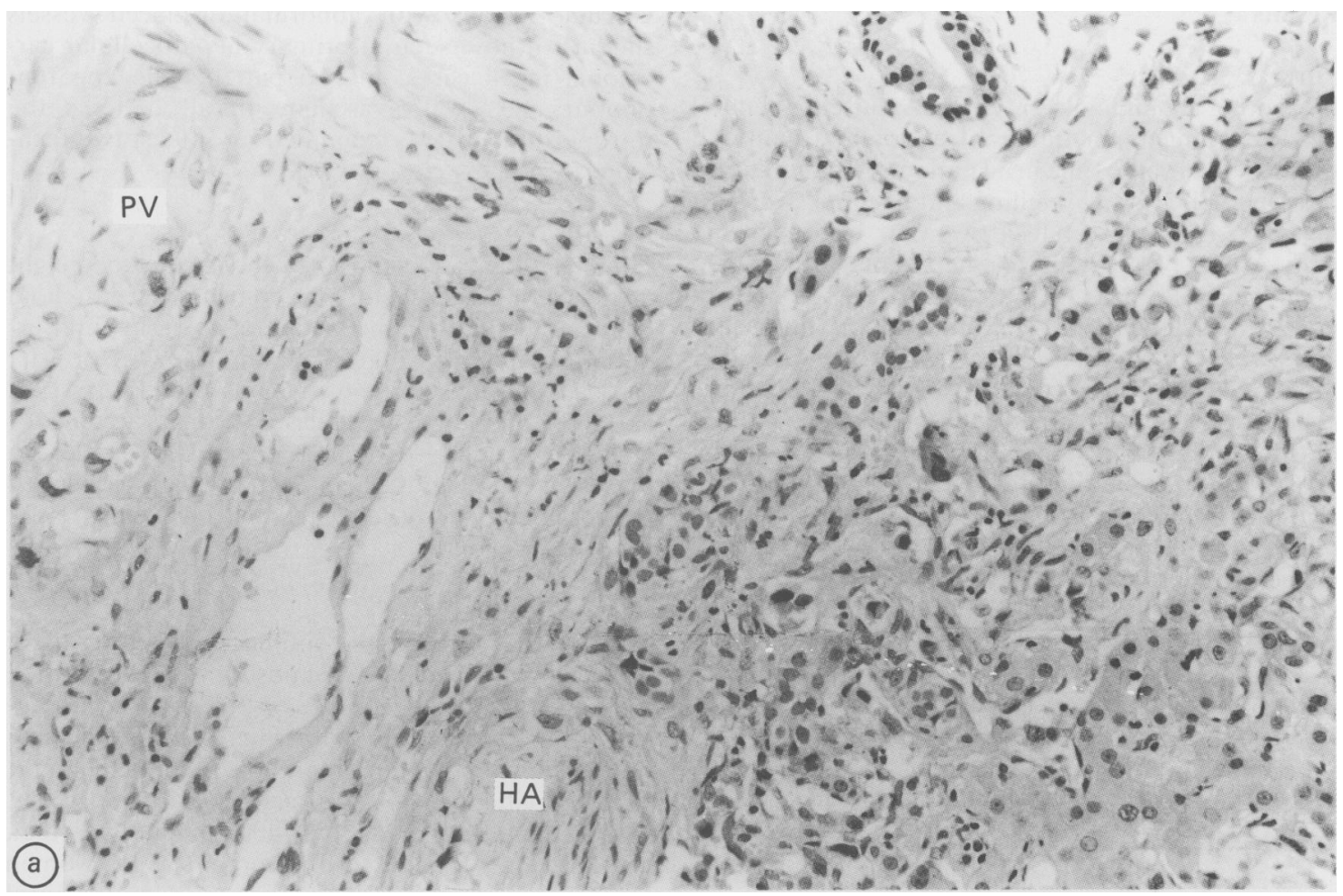

Fig. 1 (a) Liver histology from case 22. Epithelioid haemangioendothelioma cells have infiltrated parenchymal sinusoids (to the right of the field) and branches of both the portal vein (PV) and the hepatic artery $(H A)$ within a markedly enlarged portal tract. (H\&E). (b) Adjacent section stained for factor VIII associated antigen which highlights the tumour cells. (PAP method.)

investigation to have non-contiguous extrahepatic tumour. In case 3 no evidence of extrahepatic disease was found at referral or at necropsy but late presentation of metastasis from a previous colonic leiomyosarcoma was thought most likely. In two patients (cases 14 and 18), investigation of prominent non-hepatic symptoms led to the discovery of probable primary extrahepatic tumour - hip pain investigated by skeletal radiography and bone biopsy in one, and persisting nausea and epigastric discomfort investigated by endoscopy in the other (Table 1). It should be noted that earlier investigations, including laparotomy in the latter, had been inconclusive.

Table 3 Diagnostic success for retroperitoneal tumours

Patient Urography Ultrasomend Angiography CT Laparotomy

\begin{tabular}{|c|c|c|c|c|}
\hline 5 & - & & - & + \\
\hline$\delta^{\prime}$ & + & - & & + \\
\hline ) & - & & + & + \\
\hline 13 & & + & & + \\
\hline 15 & - & + & & + \\
\hline 20 & - & - & $?$ & + \\
\hline
\end{tabular}

Retroperitoneal (including inferior vena caval) tumour in six patients was revealed only by specific investigation of the area, unprompted by symptoms or signs. Single techniques often gave misleading results, and although no false positives were recorded, computed tomography scanning was the only method with no false negatives (Table 3) laparotomy having failed to show vena caval tumour in case 2 .

Clinical features (apart from those in the two cases mentioned above) and routine laboratory investigations were unhelpful in differentiating primary from secondary hepatic sarcoma, although the duration of symptoms before diagnosis was significantly longer for the group with extrahepatic diseases (median of four months, range 1-12, compared with two months, range $0 \cdot 5-8, p<0 \cdot 05)($ Mann-Whitney $U)$. Cirrhosis coexisted only in primary angiosarcomas and in only one of the three cases where it existed was it strongly suspected before examination of liver biopsy. The presence of multiple lesions on hepatic scanning was associated with a diagnosis of secondary sarcoma (seven of 10 cases); single lesions were more likely to be primary (nine of 15 cases). 


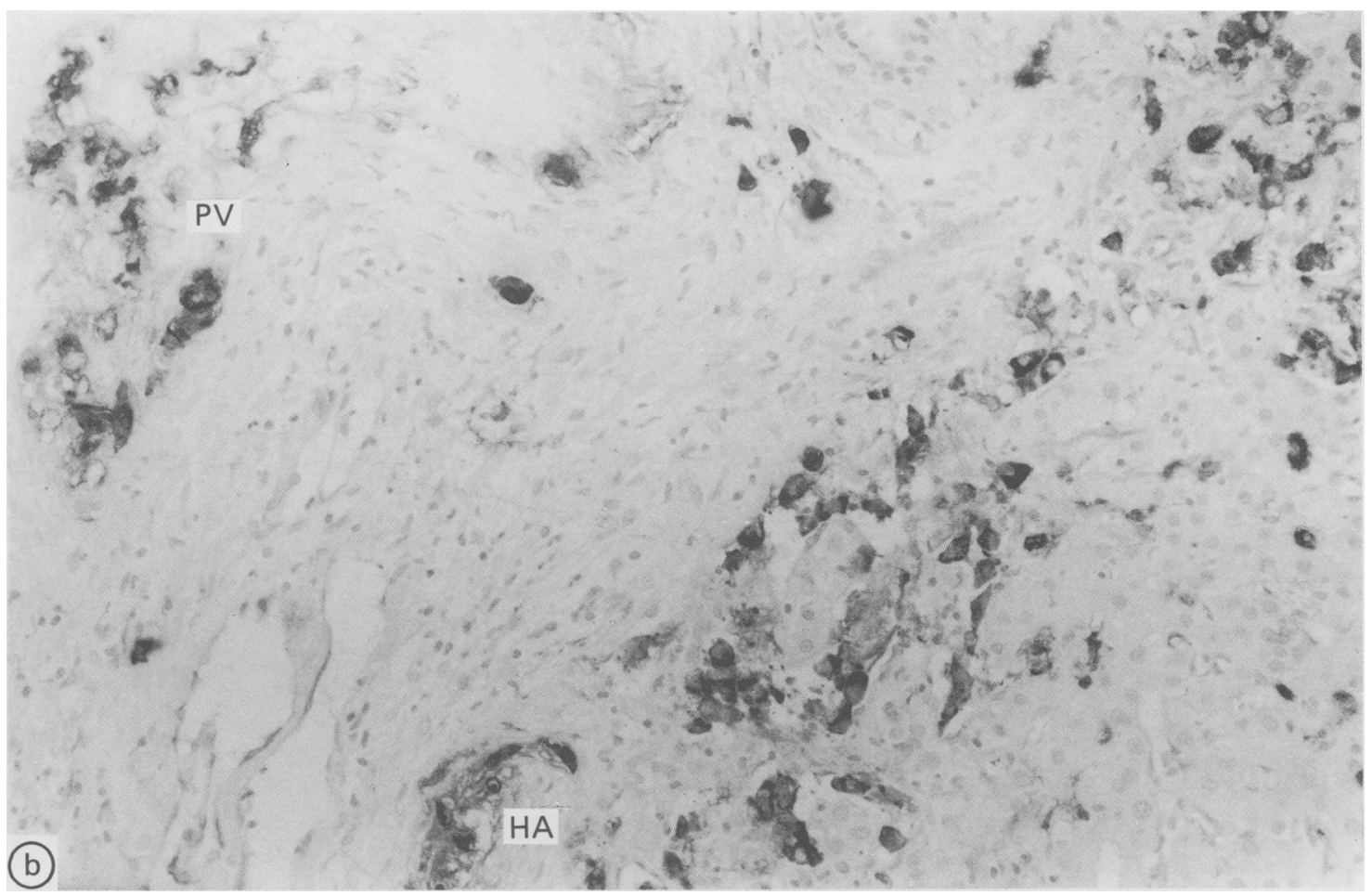

The histological distribution of the two groups proved distinct (Table 1), all angiosarcomas and undifferentiated tumours having their origin in the liver, patients with EHAE having either primary or secondary hepatic lesions, and all other differentiated tumours being of extrahepatic origin.

\section{TREATMENT AND PROGNOSIS}

\section{Primary hepatic sarcoma}

Among the 15 patients with primary hepatic sarcoma local disease extension (cases $21,22,23$, and 25) or very rapid deterioration and death (cases 1, 4, 10, 12, 17 , and 19) prevented use of surgery or other definitive management. Orthotopic liver transplantation proved possible in four cases (cases $6,7,16$, and 24); of these the three earlier patients died from perioperative problems at days 1,3 , and 25 , but the most recent case has done well and at 17 months is fit with no evidence of tumour recurrence. Case 11 with disease limited to the liver too extensive for resection and, at 69 years, above the then current upper age limit for transplantation was treated by hepatic radiotherapy. This produced satisfactory relief of pain but there was no objective evidence of tumour response, death occurring at 10 months. Hepatic artery embolisation was performed in cases 21 and 23 ; the latter died from hepatic failure at three months, but the former remains well at eight months. The remaining patients received chemotherapy. All regimes included doxorubicin; single agent therapy with the alkylating agent ifosfamide has been used recently for unresponsive disease, prompted by its successful use for other sarcomas. ${ }^{\text {1" }}$ Chemotherapy which included doxorubicin and methotrexate was followed by a reduction in tumour size (on computed tomography scan) and by a moderate improvement in liver function tests in case 22 , but this lasted only five months before rapid disease progression with the development of pulmonary metastases. Subsequently he has been treated with ifosfamide (with mesna for urothelial protection); and has had documented stable disease over the succeeding eight months and remains well. In no other case of primary hepatic sarcoma has chemotherapy yielded objective improvement. Survival for patients treated without surgery has a minimum median value of 1.75 months.

\section{Secondary hepatic sarcoma}

Patients with secondary hepatic sarcoma were treated with combination chemotherapy except for case 1.3 who obtained pain relief from hepatic irradiation, case 15 who received cisplatinum as a single agent, and case 3 who had a partial hepatectomy (in the absence of detectable disease elsewhere). He survived 


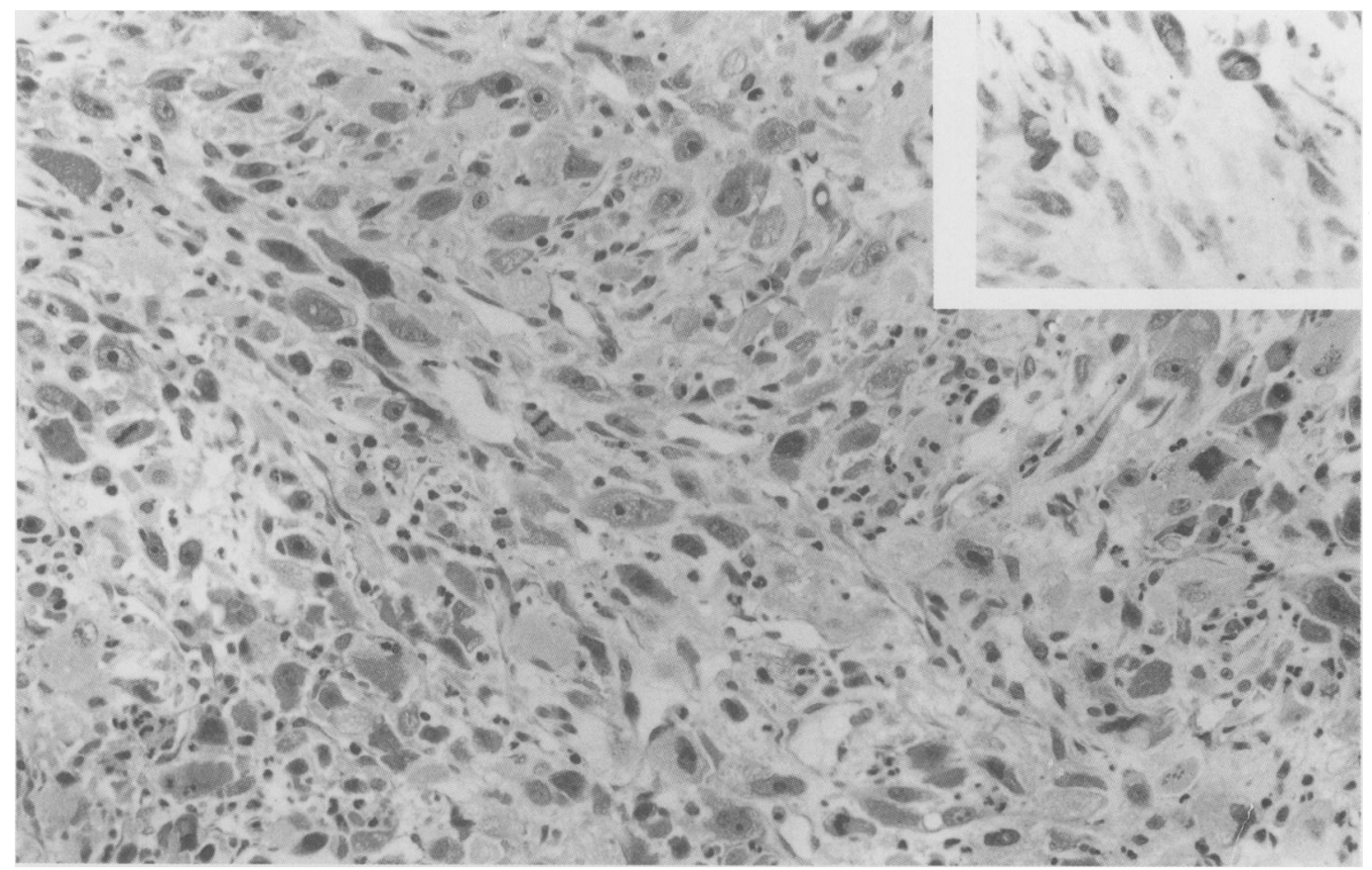

Fig. 2 Liver histology from case 17 showing an undifferentiated, pleomorphic cell tumour ( $H \& E$ ). The positive staining for vimentin (inset) provides supportive evidence for the sarcomatous nature of the lesion.

32 months but had progressive recurrent disease during the last four of these. No objective responses were documented in the remainder but case 14 had stable disease at 27 months (on doxorubicin, vincristine and dacarbazine). Median survival for this group was 10 months.

There was a significantly shorter minimum survival for those with primary hepatic sarcomas than for those with hepatic secondary deposits $(p<0 \cdot() 5)$ (Mann-Whitney U) whether or not patients treated surgically are included. If the patients with epithelioid haemangioendothelioma are considered separately and survival for the three groups. EHAE, other primary tumours, and other secondary tumours (treated conservatively) examined, median minimum survivals were $11.5,0.75$ and eight months respectively (the longer survival for patients with EHAE compared with the 'other primaries' group being significant at the $5 \%$ level).

\section{Discussion}

The present data indicate that in the clinical context of a sarcomatous tumour apparently limited to the liver, differentiation of a primary from a secondary lesion may present difficulties. The histological appearances are an important guide; angiosarcomas almost certainly have an origin within the liver and undifferentiated sarcomas usually so. Classification of the liver disease as secondary where noncontiguous extrahepatic tumour was shown on investigation can be justified on a number of grounds. Empirically, extrahepatic primary sarcoma is the more likely diagnosis as it is less rare than primary hepatic sarcoma. ' 'Spread of sarcoma is usually local, to nodes, and then through the bloodstream:" it is therefore unlikely that primary hepatic sarcoma should metastasise to a single distant site without involvement of (for example) local structures or the lungs, whereas the converse applies to liver metastases from primary tumours elsewhereparticularly within the abdomen. Both the occasional failure to detect retroperitoneal primaries at careful laparotomy, and the present widely different preponderance of histological subtypes between primary and secondary tumours introduce some doubt as to the verity of previous clinical reports of differentiated primary hepatic sarcomas (other than angiosarcomas). The relative rarity of undifferentiated primary hepatic sarcoma in the adult literature:" may 
reflect previous difficulties in distinction from other undifferentiated liver tumours; the availability of appropriate monoclonal antibody staining techniques should allow more complete histological diagnoses, the present data indicating that the frequency is likely to be similar to that of angiosarcoma.

The coexistence of primary sarcoma and cirrhosis of the liver is well recognised but it is unlikely that the frequency is as great as the $29 \%$ described by Jaffe in $1924{ }^{\prime 2}$ the present data support those, for example, of Ishak and Sussmann ${ }^{2:}$ where the association has usually been with angiosarcoma. The frequent absence of clinical features of cirrhosis emphasises the importance of careful histological assessment of tissue surrounding the tumour as well as the lesion itself.

The longer duration of symptoms before diagnosis in patients with secondary tumours matches their slower subsequent progression and presumably reflects a relatively lower grade of malignancy for these tumours. This offers potential prognostic value in individual patients at the time of diagnosis. Exploratory laparotomy is not always reliable in assessment of the retroperitoneum - the commonest site for occult primaries - and its diagnostic use can no longer be recommended. Of the other techniques currently available computed tomography scanning yielded no false negative and should be the investigation of choice.

Results from the present study indicate that extensive investigation of the skeleton and gastrointestinal tract is only necessary in patients with symptoms attributable to these systems or where resection is contemplated; it does not appear helpful in differentiation of primary from secondary hepatic lesions.

Epithelioid haemangioendothelioma has been recognised only recently" and two of the present patients were correctly classified only retrospectively. The previous histological diagnoses, (undifferentiated spindle cell sarcoma) were felt unsatisfactory but the best compromise available at the time. Our data like those of Ishak et $a^{\prime \prime}$ indicate that these tumours have a better prognosis than other hepatic sarcomas; perhaps because all of our patients were symptomatic their median survival was less than that for Ishak's group. The metastasising potential of EHAE is confirmed, and there was no obvious difference in prognosis between those presenting with primary from those with secondary hepatic disease.

The patient with a previously resected benign leiomyoma' (case 3 ) deserves comment in view of the classification of his subsequent leiomyosarcoma as an hepatic secondary. Although progression from benign to malignant mesenchymal tumour has been described" even a four year disease free interval is quite consistent with the original tumour also having been of very low grade malignancy; complete excision of the original tumour and the absence of micrometastases cannot be proven.

The present series contains a similar prevalence of smokers to control populations and this is unlikely to be of major aetiological importance. Prolonged use of the oral contraceptive was a potential risk factor in four of our patients - that is, one third of the women included here and about half of those in whom the reproductive period coincided with availability of the pill - two of whom had EHAE; the data for other malignant liver tumours ${ }^{1+}$ and a recent report linking EHAE with pill usage (five of five cases) ${ }^{1 *}$ suggest an increased risk in these subjects. The present frequency of a past history of malaria appears greater than would be expected by chance (and Ishak records this in another patient with angiosarcoma ${ }^{2}$ ); available data from tropical areas do not assist in this respect. The presumed causal association of vinyl chloride and angiosarcoma in case 19 is interesting in view of the documented very short exposure, but also in being the only one of eight angiosarcomas where a recognised aetiological factor was present. Radical excision - resection or liver transplantation according to the extent of spread within the liver - offers the only hope of cure" and should be the aim in primary hepatic sarcoma. Where surgery is contraindicated, as is likely in the majority of patients, active therapy (embolisation/chemotherapy/radiotherapy) will usually be considered, but results compare poorly with those for other sarcomas ${ }^{17-21}$ not least because of the very rapidly progressive disease in some patients. Radiotherapy or embolisation may be most valuable where hepatic pain is prominent as for other liver tumours. The most efficacious chemotherapeutic agents for primary hepatic sarcomas have not been established, and no data indicate improved survival for patients so treated. First line therapy with ifosfamide and mesna given every three weeks would appear reasonable, possibly with the addition of doxorubicin. With the probable exception of angiosarcomas rarity is likely to preclude adequate controlled trials even on a multicentre basis.

We thank Mr P Aldis for technical help with the histological preparations. Mr R Jenkins for the microphotographs and are grateful to our colleagues at other hospitals for referring the patients.

\section{References}

1 Chang WWL. Agha FP. Morgan WS. Primary sarcoma of the liver in the adult. (ancer. 1983: 51: 1510-7.

2 Ishak KG. Mesenchymal tumours of the liver. In: 
Okuda K, Peters RL, eds. Hepatocellular carcinoma. New York: Wiley 1976: 247-307.

3 Stocker JT, Ishak KG. Undifferentiated (embryonal) sarcoma of the liver. Report of 31 cases. Cancer 1978; 42: $336-48$.

4 MacMahon HE, Murphy AS, Bates MI. Endothelial cell sarcoma of liver following thorotrast injections. Am J Pathol 1947; 23: 585-611.

5 Regelson W, Kim U, Ospina J, et al. Hacmangioendothelial sarcoma of liver from chronic arsenic intoxication. Cancer 1968; 21: 514-22.

6 Makk L, Delmore F, Creech JL, et al. Clinical and morphological features of hepatic angiosarcoma in vinyl chloride workers. Cancer 1976; 37: 149-63.

7 Stout AP, Lattes R. Tumors of the soft tissues. Atlas of tumor pathology. Second series, fascicle 1. Washington, DC: Armed Forces Institute of Pathology, 1967.

8 Miettinen M, Lehto V-P, Virtanen I. Antibodies to intermediate filament proteins in the diagnosis and classification of human tumours. Ultrastruct Pathol 1984; 7: 83-107.

9 Ishak KG, Sesterhenn IA, Goodman ZD, Rabin L, Stromeyer FW. Epithelioid hemangioendothelioma of the liver: a clinicopathologic and follow-up study of 32 cases. Human Pathol 1984; 15: 839-52.

10) Stuart-Harris RC, Harper PG, Parsons CA, et al. High dose alkylation therapy using ifosfamide with mesna in the treatment of adult advanced soft-tissue sarcoma. Cancer Chemother Pharmacol 1983; 11: 69-72.

11 Lee Y-TNM. Leiomyosarcoma of the gastrointestinal tract: general pattern of metastasis and recurrence. Cancer Treat Rev 1983; 10: 91-101.

$12 \mathrm{Jaffe}$ RH. Sarcoma and carcinoma of the liver following cirrhosis. Arch Intern Med 1924; 33: 330-42.

13 Sussman EB, Nydick I, Gray GF. Haemangioendothelial sarcoma of the liver and hemochromatosis. Arch Pathol 1974; 97: 39-42.

14 Neuberger J, Forman D, Doll R, Williams R. Oral contraceptive usage and hepatocellular carcinoma. Br Med J 1986; 292: 1355-7.

15 Dean PJ, Haggitt RC, O’Hara CJ. Malignant epithelioid hemangioendothelioma of the liver in young women. Am J Surg Pathol 1986; 9: 695-704.

16 Chen KTK. Hepatic leiomyosarcoma. J Surg Oncol 1983; 24: 325-8.

17 Das Gupta TK, Patel MK, Chaudhuri PK, Briele HA. The role of chemotherapy as an adjuvant to surgery in the initial treatment of primary soft tissue sarcomas in adults. J Surg Oncol 1982; 19: 139-44.

18 Bui NB, Chauvergne J, Hocke C, Durand M, Brunet R, Coindre J-M. Analysis of a series of 60 soft tissue sarcomas in adults treated with a cyclophosphamidevincristine-adriamycin-dacarbazine (CYVADIC) combination. Cancer Chemother Pharmacol 1985; 15: 82-5.

19 Suit HD, Mankin HJ, Wood WC, Proppe KH. Preoperative, intraoperative, and postoperative radiation in the treatment of primary soft tissue sarcoma. Cancer 1985; 55: 2659-67.

20) Anonymous. Osteosarcoma [Editorial]. Lancet 1985; 2: $131-3$. 